\section{Preventing cardiovascular disease}

\section{in diabetes}

John N Harvey MD FRCP, Senior Lecturer in Diabetes, Endocrinology and Metabolism, University of Wales College of Medicine

Clin Med 2004;4:311-4

\section{Epidemiology}

People with type 1 and type 2 diabetes have a higher relative risk of coronary heart disease (CHD) although, because they are younger, the absolute numbers in type 1 patients remain small. The absolute risk of myocardial infarction (MI), stroke or cardiovascular death in type 2 diabetic patients is as high as in non-diabetic individuals with symptomatic coronary disease, a group targeted for secondary prevention., ${ }^{1,2}$ Moreover, diabetes worsens outcomes in acute coronary syndromes, with a five-year mortality of at least $50 \% .^{3}$ Thus, people with type 2 diabetes are prime candidates for primary or secondary prevention. The growing prevalence of diagnosed type 2 diabetes makes it an ever increasing cause of cardiovascular morbidity and mortality, particularly in ethnic groups where diabetes is more common (eg Indo-Asian and Black people). Identification of cases of impaired glucose tolerance (IGT) and asymptomatic type 2 diabetes, as recommended in the Diabetes National Service
Framework (NSF), will yet further increase the number of candidates for prevention.

\section{Risk factors and intervention based on them}

In patients without symptoms, intervention to prevent cardiovascular disease is based on assessment of risk. The various risk factors have an additive or even multiplicative effect on mortality, such that each should be addressed where possible. ${ }^{4}$ To determine which interventions are beneficial we must look to prospective intervention trials.

\section{Obesity}

Obesity is clearly linked to type 2 diabetes and CHD. There is only weak evidence that dietary advice can prevent coronary events in the population and reductions in cholesterol are small and ill sustained. However, intensive diet and exercise programmes can prevent the development of type 2 diabetes in the obese. ${ }^{5,6}$ A sustained reduction in body weight substantially improves glycaemic control, reduces blood pressure and improves well-being, but only a minority achieve it. Dietary advice at first diagnosis of diabetes should include avoidance of simple sugars to improve glycaemia and reduction in alcohol

\section{Key Points}

Cardiovascular disease, especially coronary heart disease (CHD), is the main cause of mortality and morbidity in type 2 diabetes

Major trials have shown that a number of interventions are highly effective in reducing major outcomes including death; their effects are additive

Lipid lowering, reduction of blood pressure, aspirin therapy and stopping smoking are the most important preventive measures

There is a close link between all degrees of proteinuria and CHD in diabetes

KEY WORDS: aspirin, cardiovascular disease, hypertension, lipid lowering therapy, type 2 diabetes intake because of its high calorie content. Low carbohydrate (Atkins) diets may prove better than conventional dietary recommendations but so far studies have been only short term. ${ }^{7,8}$

\section{Smoking}

Smoking greatly increases the risk of both micro- and macrovascular disease; stopping smoking is at least as beneficial to macrovascular outcomes as any other intervention discussed in this review. ${ }^{9}$ However, the number of patients who give up smoking after advice programmes remains low.

\section{Exercise}

Exercise lowers glucose levels and raises insulin sensitivity. It improves the lipid profile, blood pressure, fitness and well-being independently of any effect on weight and glycaemia. However, the benefits wear off within 72 hours of the last session and people with long duration diabetes and on insulin seem to benefit less. Therefore, exercise should be frequent and start early, be prescribed individually and followed up. Some diabetes units have collaborated with local fitness centres, while others have provided initial supervision and then monitored patients' subsequent home exercise.

\section{Hyperglycaemia}

The UKPDS showed that hyperglycaemia increases the risk of $\mathrm{CHD}$, peripheral and cerebrovascular disease (see end of text for explanation of trial acronyms). However, improving glycaemic control with insulin or sulphonylurea had only marginal macrovascular benefit. Likewise, there is little evidence that improving glycaemia reduces coronary events in type 1 diabetes. Smaller follow-up studies in type 2 diabetes have, like UKPDS, shown a clear effect on microvascular end points but no significant effect on CHD. People with IGT also have a substantially higher risk of cardiovascular events, ${ }^{10}$ implying that either slight hyperglycaemia damages large vessels or responsibility lies with other 
components of the metabolic syndrome (see below). Active treatment with diet, exercise, aspirin, antihypertensive and lipid lowering therapy is indicated in IGT, as in type 2 diabetes. Metformin may also be used. ${ }^{6}$

\section{Hyperlipidaemia}

Type 2 diabetic patients have an atherogenic lipid profile with raised levels of small dense low-density lipoprotein (LDL) cholesterol, low high-density lipoprotein (HDL) cholesterol and raised triglycerides. Oxidation and glycation of LDL accelerates its uptake by scavenger macrophages. Once these cells are lipid laden, they become the foam cells that develop into atheromatous plaques. Low HDL cholesterol levels are an independent risk factor for cardiovascular events. ${ }^{4}$ HDL may also be functionally deficient in diabetes because it does not protect LDL from oxidation as well as in non-diabetic individuals. ${ }^{1}$ Intervention studies have provided limited diabetesspecific data, but in the HPS, which included more than 4,000 diabetic subjects, simvastatin $40 \mathrm{mg}$ reduced the risk of acute coronary events, stroke or revascularisation by about $25 \%$ irrespective of baseline cholesterol level. ${ }^{11}$ Such 'pleiotropic' effects of statins (benefit unrelated to cholesterol lowering) have led to the suggestion that statin therapy be used in type 2 diabetes irrespective of cholesterol level because risk is so high. Fibrates are also effective, perhaps by elevating HDL cholesterol - in the VA-HIT study gemfibrozil reduced major cardiovascular events by $32 \% .^{12}$

Risk tables are widely used for decisions on lipid lowering therapy but must be used with caution because the Framingham cohort, on which most are based, included only small numbers of diabetic patients and had a limited ethnic and socio-economic composition. Young type 2 patients are another special group because their five- or 10-year risk is low but there is a case for early intervention (as discussed elsewhere). ${ }^{13} \mathrm{~A}$ risk calculator based on the UKPDS for newly diagnosed diabetic patients is now available.

\section{Hypertension}

Hypertension is common in diabetes; its greatest impact is on microvascular, cerebrovascular and heart failure end points, with less effect on MI and peripheral vascular disease. Tight control of hypertension in UKPDS reduced the risk of macrovascular outcomes, particularly stroke and congestive heart failure (CCF) but the reduction in MI was not significant. Atenolol and captopril were equally effective. The ABCD, FACET and HOPE trials all showed that angiotensinconverting enzyme inhibitors (ACEIs) prevent cardiovascular events in diabetes. An analysis of 45,000 patients showed that beta-blockers also prevent MI. ${ }^{14}$ ACEIs are well tolerated and angiotensin II receptor antagonists (AIIRAs), introduced since these trials, may be combined with them.

\section{TRIAL ACRONYMS}

$\begin{array}{ll}\text { 4S } & \text { Scandinavian Simvastatin Survival Study } \\ \text { ABCD } & \text { Appropriate Blood Pressure Control in Diabetes } \\ \text { ALLHAT } & \begin{array}{l}\text { Antihypertensive and Lipid-Lowering Treatment to Prevent } \\ \text { Heart Attack Trial }\end{array} \\ \text { DCCT } & \text { Diabetes Control and Complications Trial (US) } \\ \text { FACET } & \text { Fosinopril versus Amlodipine Cardiovascular Events Trial } \\ \text { HOPE } & \text { Heart Outcomes Prevention Evaluation } \\ \text { HOT } & \text { Hypertension Optimal Treatment } \\ \text { HPS } & \text { Heart Protection Study } \\ \text { UKPDS } & \text { UK Prospective Diabetes Study } \\ \text { VA-HIT } & \text { Veterans Affairs High-Density Lipoprotein Intervention Trial }\end{array}$

The importance of checking renal function after initiating either class of drug cannot be overemphasised. Renovascular disease is common in type 2 diabetes. Doxazosin increased the risk of CCF in the large ALLHAT trial, so is not recommended for first-line use. Hypertension associated with microalbuminuria or established nephropathy should be treated as volume expanded (renal) hypertension; loop diuretics are often required.

\section{Microalbuminuria}

A small increase in urinary protein excretion (microalbuminuria) is a precursor to renal failure from diabetic nephropathy and indicates a high risk of CHD and premature death. This risk increases in parallel with the degree of proteinuria. While increasing numbers of type 2 patients are going on to dialysis, they have poor outcome because of cardiovascular disease. Therefore, the dual aim in proteinuric patients is to prevent cardiovascular events and preserve renal function. ACEIs and AIIRAs both reduce progression of microalbuminuria to established nephropathy, retard deterioration of renal function and improve survival (combined end point) in patients with heavy proteinuria. It is less clear whether AIIRAs influence cardiovascular death.

Microalbuminuria has other causes, including urine infection and most renal and renal tract pathologies, pyrexia, recent exertion and uncontrolled heart failure.

\section{Left ventricular hypertrophy}

Left ventricular hypertrophy, an independent predictor of coronary events, has been used as an end point in some studies.

\section{Haemostatic factors}

Circulating levels of fibrinogen and other coagulation factors are raised in diabetes. Platelets from diabetic subjects show increased adhesiveness, mediated in part by fibrinogen binding to the glycoprotein IIb/IIIa complex. Fibrinolytic activity is often suppressed via the 
inhibitory actions of plasminogen activator inhibitor, $90 \%$ of which is carried in vivo by platelets.

Aspirin inhibits platelet function and reduces the risk of major cardiovascular events by about $25 \%$ in diabetic patients. The American Diabetes Association recommends low dose aspirin therapy for primary prevention in diabetic patients from age $30 .{ }^{15}$ Some may feel the incidence of MI in the UK does not justify such a young start age although the HOT trial confirmed that such therapy is relatively safe.

\section{Metabolic syndrome}

The term 'metabolic syndrome' refers to a group of related disorders in which insulin resistance is combined with one or more of the following features: obesity, type 2 diabetes, essential hypertension, hypertriglyceridaemia, low HDL cholesterol and microalbuminuria.

This concept has altered our perception of type 2 diabetes from a primary disorder to a syndrome with insulin resistance the central feature and a major impact on the vasculature. It remains unclear whether insulin insensitivity and hyperinsulinism directly cause atherosclerosis independently of glycaemia, perhaps via endothelial dysfunction.

\section{Hormone replacement therapy}

Postmenopausal women on oestrogen therapy had reduced CHD risk in observational studies but no benefit in a prospec- tive intervention trial. It is no longer recommended for CHD prophylaxis.

\section{Secondary prevention of cardiovascular disease in diabetes}

In the $4 \mathrm{~S}$ study in patients with established CHD, the six-year event rate in the diabetic subgroup was as high as $49 \%$ despite a baseline total cholesterol of only $5.5 \mathrm{mmol} / \mathrm{l}$. Thus, all cardiovascular risk factors need to be actively targeted in this group of patients. In addition, silent CHD is more common in diabetes than in the general population, mainly as a result of cardiac autonomic neuropathy. Its prognosis is poor, as for overt disease. This blurs the distinction between primary and secondary prevention.

\section{Conclusions}

Type 2 diabetes is a multifaceted disorder in which the leading cause of mortality and morbidity is large vessel disease, especially CHD. The focus is now firmly on cardiovascular disease prevention. Aggressive management of lipids and blood pressure, wider use of aspirin, ACEIs and AIIRAs together with lifestyle change are now seen to be at least as important as glycaemic control. Expert groups have published increasingly strict targets for the various parameters (Table 1) as larger trials have shown the benefits of treating lesser degrees of abnormality. There is also a move towards earlier intervention.

Table 1. Targets for glycaemic control, lipid therapy and blood pressure (BP). BP recommendations from the recent British Hypertension Society guidelines recommending lower total and low-density lipoprotein (LDL) cholesterol levels in hypertensive diabetic patients. ${ }^{16}$

\begin{tabular}{ll} 
Parameter & \multicolumn{1}{c}{ Target } \\
\hline Glycaemia in type 1 diabetes (DCCT) & $\mathrm{HbA}_{1 \mathrm{c}} 7.0 \%$ \\
Glycaemia in type 2 diabetes (UKPDS) & $\mathrm{HbA}_{1 \mathrm{c}} 7.0 \%$ \\
Blood pressure & $\leq 130 / 80 \mathrm{mmHg}$ \\
& audit standard $\leq 140 / 80 \mathrm{mmHg}$ \\
Cholesterol: & $<5 \mathrm{mmol} / \mathrm{l}$ \\
- total & $<2.6 \mathrm{mmol} / \mathrm{l}$ \\
- LDL & $>1.1 \mathrm{mmol} / \mathrm{l}$ \\
- HDL & $<2.0 \mathrm{mmol} / \mathrm{l}$ \\
\hline Triglycerides & \\
\hline
\end{tabular}

$\mathrm{HbA}_{1 \mathrm{c}}=$ glycated haemoglobin; $\mathrm{HDL}=$ high-density lipoprotein.

The NSF recommends more routine checks in primary care and screening for type 2 diabetes. The increasing prevalence of diabetes and lower threshold for diagnosis will provide increasing justification for this in terms of numbers of new cases identified. Earlier intervention is expected to improve outcome.

\section{Conflict of interest}

None.

\section{References}

1 Beckman JA, Creager MA, Libby P. Diabetes and atherosclerosis: epidemiology, pathophysiology, and management. Review. JAMA 2002;287:2570-81.

2 Haffner SM, Lehto S, Ronnemaa T, Pyorala K, Laakso M. Mortality from coronary heart disease in subjects with type 2 diabetes and in nondiabetic subjects with and without prior myocardial infarction. $N$ Engl J Med 1998;339:229-34.

3 Herlitz J, Karlson BW, Lindqvist J, Sjolin M. Rate and mode of death during five years of follow-up among patients with acute chest pain with and without a history of diabetes mellitus. Diabet Med 1998;15: 308-14.

4 Stamler J, Vaccaro O, Neaton JD, Wentworth D. Diabetes, other risk factors, and 12-yr cardiovascular mortality for men screened in the Multiple Risk Factor Intervention Trial. Diabetes Care 1993;16: 434-44.

5 Tuomilehto J, Lindstrom J, Eriksson JG, Valle TT et al. Prevention of type 2 diabetes mellitus by changes in lifestyle among subjects with impaired glucose tolerance. $N$ Engl J Med 2001;344:1343-50.

6 Knowler WC, Barrett-Connor E, Fowler SE, Hamman RF et al. Diabetes Prevention Programme Research Group. Reduction in the incidence of type 2 diabetes with lifestyle intervention or metformin. $N$ Engl J Med 2002;346:393-403.

7 Samaha FF, Iqbal NN, Seshadri P, Chicano $\mathrm{KL}$ et al. A low-carbohydrate as compared with a low-fat diet in severe obesity. $N$ Engl J Med 2003;348:2074-81.

8 Foster GD, Wyatt HR, Hill JO, McGuckin BG et al. A randomized trial of a low-carbohydrate diet for obesity. $N$ Engl J Med 2003;348:2082-90.

9 Yudkin JS. How can we best prolong life? Benefits of coronary risk factor reduction in non-diabetic and diabetic subjects. $B M J$ 1993;306:1313-8.

10 Coutinho M, Gerstein HC, Wang Y, Yusuf S The relationship between glucose and incident cardiovascular events. A metaregression analysis of published data from 
20 studies of 95,783 individuals followed for 12.4 years. Diabetes Care 1999;22: 233-40.

11 Heart Protection Study Collaborative Group. MRC/BHF Heart Protection Study of cholesterol lowering with simvastatin in 20,536 high-risk individuals: a randomised placebo-controlled trial. Lancet 2002;360: 7-22.

12 Rubins HB, Robins SJ, Collins D, Nelson DB et al. Diabetes, plasma insulin, and cardiovascular disease: subgroup analysis from the Department of Veterans Affairs high-density lipoprotein intervention trial (VA-HIT). Arch Intern Med 2002;162: 2597-604.

13 Winocour PH, Fisher M. Prediction of cardiovascular risk in people with diabetes. Review. Diabet Med 2003;20:515-27.

14 Chen J, Marciniak TA, Radford MJ, Wang Y, Krumholz HM. Beta-blocker therapy for secondary prevention of myocardial infarction in elderly diabetic patients. Results from the National Cooperative Cardiovascular Project. J Am Coll Cardiol 1999;34: 1388-94.

15 Colwell JA; American Diabetes Association. Aspirin therapy in diabetes. Diabetes Care 2003;26(Suppl 1):S87-8.

16 Williams B, Poulter NR, Brown MJ, Davis M et al. British Hypertension Society guidelines for hypertension management 2004 (BHS-IV): summary. BMJ 2004;328: 634-40.

\section{Diabetes in hospital}

\section{Arpan Bhattacharya DM MCRP,}

Consultant Endocrinologist,

Manipal Hospital, Bangalore, India

Tim Dornan DM FRCP, Consultant Physician, Hope Hospital, Salford, Manchester

\section{Clin Med 2004:4:314-7}

As diabetes is becoming more prevalent, the number of diabetic patients admitted to hospital is rising. Some admissions are directly due to diabetes but diabetic adults are also six times more likely than non-diabetic adults to be admitted for other reasons. ${ }^{1}$ This is a worldwide and large-scale problem. Up to $10 \%$ of UK hospital inpatients ${ }^{2}$ and six million people hospitalised each year in the USA are diabetic. ${ }^{3}$ Patient associations identify hospitalisation as a priority time in diabetic life for better care because poor glycaemic control increases susceptibility to complications and lengthens hospital stay. ${ }^{4}$ It is also an unhappy time if, as too often happens, patients who are experts in self-care are denied information, support and autonomy. ${ }^{5}$

\section{Why glucose control destabilises in hospital}

Stress causes an outpouring of counter-regulatory hormones including adrenaline, glucagon, cortisol and growth hormone. These accelerate catab- olism, hepatic gluconeogenesis and lipolysis, and raise blood glucose, fatty acids and ketone bodies. In acute myocardial infarction (AMI), for example, there is a direct correlation between plasma adrenaline and glucose concentrations. Hospitalised patients are physically inactive - another potent reason for hyperglycaemia. Blood glucose rises, but in an unpredictable way because other factors favour hypoglycaemia. Ill people may not be able to eat as normal and, at least in the UK, their hospital diet is often unsatisfactory. ${ }^{6}$ They may be unable to call for extra food when hypoglycaemic because they are physically incapable or cognitively impaired.

Most unsatisfactory of all, they may be 'treated' in an authoritarian way by people who know less about diabetes than they do. This was graphically illustrated by a UK trial in which a diabetes specialist nurse shortened hospital stay by $27 \%$ through supervising and coordinating diabetes care. ${ }^{7}$

\section{Does good glycaemic control really matter?}

Logically, good control might be expected to improve well-being, prevent postoperative infection, hasten the resolution of infection, facilitate wound healing and prevent severe hyperglycaemia. A turning point in hospital care was the Dextrose Insulin and Glucose in Acute Myocardial Infarction (DIGAMI) study, which showed that tight control at the time of MI and over

\section{Key Points}

\section{Up to $10 \%$ of NHS inpatients have diabetes}

It is unrealistic to expect every patient to have normal blood glucose all the time; we define good control as four out of five preprandial glucose values in the range 4-10 $\mathrm{mmol} / \mathrm{l}$

Acutely ill patients should be managed with intravenous insulin/dextrose

Patients well enough to eat should be treated with subcutaneous insulin; a four times daily 'basal-bolus' regimen reduces the glucose rise after meals and is flexible enough to be adjusted within-day to correct for any upwards or downwards trend

Analogues are showing promise as the insulins of choice for patients in hospital

KEY WORDS: Diabetes mellitus, inpatient management 